\title{
Earthquake Magnitude and Surface Fault Formation
}

\author{
By \\ Michio Otsuka \\ Department of Physics, Faculty of Science, Kumamoto University
}

\section{§1. Introduction}

When a fault is formed on the earth's surface in connection with the occurrence of an earthquake, its length and direction are known to be generally concordant with the magnitude and origin mechanism of the pertaining earthquake. K. IIDA (1959) studied the relation between the length of fault and the magnitude of the pertaining earthquake. In Fig. 1, which was taken from his paper, the fault length $L(\mathrm{~km})$ is plotted in the logarithmic scale against the corresponding earthquake magnitude $M$. IIDA expressed the relation by

$$
M=(6.27 \pm 0.14)+(0.63 \pm 0.09) \log L .
$$

Combining eq. (1) with Gutenberg-Richter's formula of earthquake energy $E$ :

$$
\log E=11.8+1.5 M
$$

he obtained the relation

$$
E=1.6 \times 10^{21} \times L^{0.9}
$$

Seeing that the exponent of $L$ in eq. (3) is nearly equal to unity, IIDA concluded that the fault length is approximately proportional to the energy of the pertaining earthquake. A similar relation was obtained by $D$. Tocher (1958) for earthquakes in California and Nevada.

Apart from the problem of earthquake fault, the concept of earthquake source volume was proposed by $\mathrm{C}$.' Tsusor which has proved itself to be useful for elucidating the dependence of aftershock area (Tsubor, 1956) and the duration of energy radiation (Tsubor and OtsuKa, 1963) on earthquake magnitude.

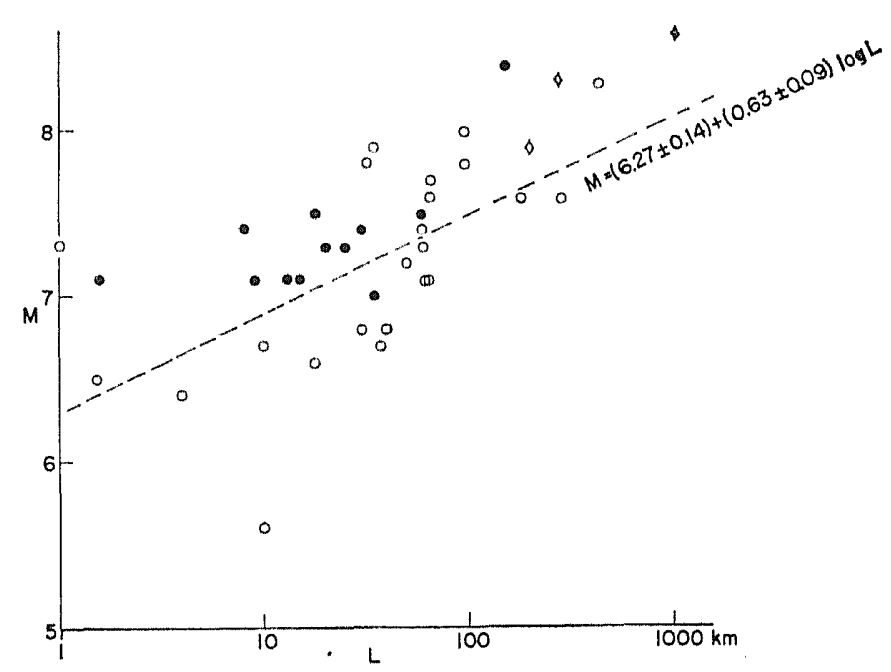

Fig. 1. Relation between earthquake magnitude $M$ and length of earthquake fault $L$. (after K. IIDA (1959)), data in the vicinity of Japan, $\bigcirc$ : data all over the world, $\diamond$ : subsequent data not included in IIDA paper. (faults which accompanied the Outer Mongolian (1957), Alaskan (1958), and Chilean (1960) earthquake.) 
What takes place within an earthquake source volume when the energy stored in it is radiated across its surface is not clearly known, but very probably this is some kind of fracture formation. In the following study, an attempt will be made to interpret the relation between the fault length and the earthquake magnitude on the basis of the geometrical situation of the source volume with respect to the earth's crust.

\section{§2. Upper limit of fault length $\mathrm{Lm}$}

In Fig. 2, the same points are plotted as in Fig. 1, the only difference of the two being that, while in Fig. 1, a straight line is drawn to represent the overall distribution of points, two lines are drawn in Fig. 2 which mark the limits of point scattering. The maximum fault length $L m$ for earthquakes of magnitude $M$ increases with increasing $M$ as shown by the dashed line in Fig. 2. The line is expressed by

$$
M=2 \log L m-6.4 \text {. }
$$

The six points for larger $M$ are excluded for deducing this formula. These will be referred to in $\S 4$.

The fault length of each individual earth- quake must depend on various factors, such as its energy, focal depth, mechanism at the origin, as well as the strength of the medium from which it originates. But an upper limit of fault length must exist for each magnitude, because the strain energy accumulation per unit volume of the material of the earth's crust is more or less invariable and therefore the site of storage of a limited amount of energy corresponding to that magnitude cannot be indefinitely extended. If $L m$ is identified with the maximum linear dimension of the source volume of an earthquake of magnitude $M$, the relation between $L m$ and $E$ will be obtained by eliminating $M$ from eq. (2) and eq. (4), thus

$$
\log E=2.2+3 \log L m
$$

or

$$
E=150 \times \mathrm{Lm}^{3} .
$$

Eq. (5) is quite reasonable in that the exponent of $\mathrm{Lm}$ is 3, agreeing with the concept of voluminal storage of earthquake energy. But the numerical coefficient which is 150 is much smaller than that expected from the earthquake source volume theory. According to Tsubor, the maximum strain energy which

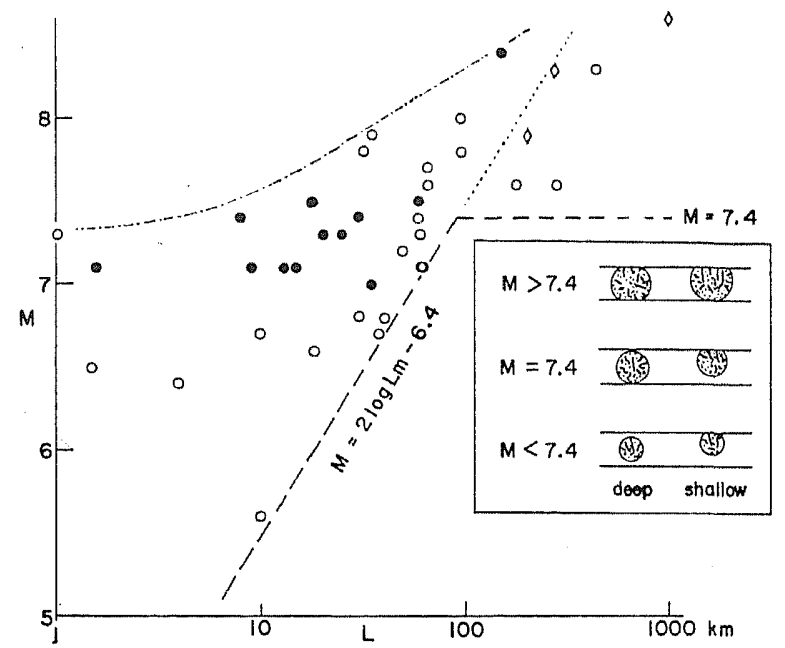

Fig. 2. Relation between earthquake magnitude $M$ and length of earthquake fault $L$. Dashed and chain lines show the upper and lower boundaries of fault length respectively. Inset shows the geometrical situation of focal region with respect to the earth's crust. 
can be stored up per unit volume of the material of the earth's crust is $3 \times 10^{3} \sim 2 \times 10^{4}$ ergs. Tsubor obtained these values from the study of deformations of the earth's crust as disclosed by geodetic measurements, especially of those which took place in connection with fault-producing earthquakes. With these values of Tsubor and with the assumptions that the earthquake source volume is spherical in shape and that the maximum fault length $\mathrm{Lm}$ represents the diameter of the sphere, this numerical coefficient should be 1,500 10,000 which is 10 60 times as large as that in eq. (5). In other words, observed $L m$ is $2 \sim 4$ times too long in comparison with the length expected from the mechanical strength of the earth's crust based on crustal deformation data. This discrepancy may be partly due to the departure of the shape of an earthquake source volume from a sphere, but what seems to be more important is that while GutensergRichTER's energy formula (2) which was used in deriving eq. (5) is based on observations of seismic waves, Tsubor's estimation is on crustal deformation data. It may be doubted if the seismic wave energy and the crustal deformation energy can be regarded to be about the same, although TsuBor has succeeded in elucidating various earthquake phenomena on that supposition.

\section{§3. Probability of fault appearance}

Let us consider the geometrical situation of an earthquake source volume with respect to the earth's crust. If the source volume is situated deep enough beneath the earth's surface so that it is entirely merged within the earth's crust, no fracture can be observed on the surface of the earth, while if the source volume is situated so shallow that a part of the volume emerges on the earth's surface, fracture will be seen. In this way, there is a possibitity of explaining, in a more or less quantitative way, the empirical fact that shallow and large earthquakes are more often accompanied by visible fault than deep and small ones are. The scatter of points in Figs. 1 and 2 may also be explained by the diversity of the dimension and depth of earthquake source volume in relation to the thickness of the earth's crust.

For simplicity, the following two assumptions are made:

(A) the earthquake source volume is spherical in shape with radius $a$

and

(B) the occurrence of fracture is restricted within the earth's crust of thickness $H$.

From (B), it follows that the depth $d$ of the centre of source sphere must satisfy the following condition:

$$
d<H-a .
$$

If the condition

$$
d<a
$$

is satisfied, then fault will appear on the earth's surface. If earthquakes occur evenly through all depths within the earth's crust satisfying condition (6), the probability $p^{\prime}(a)$ that a given earthquake having the source volume of radius $a$ is accompanied by surface fault appearance is expressed simply by

$$
p^{\prime}(a)=\frac{N_{f}^{\prime}(a)}{N^{\prime}(a)}=\frac{a}{H-a},
$$

where $N^{\prime}(a)$ and $N_{f}{ }^{\prime}(a)$ are the total number of earthquakes and the number of earthquakes accompanied by faults respectively, all having the focal radius $a$. $a$ is related to the earthquake magnitude $M$, so that $a$ in eq. (8) can be replaced by $M$ as follows:

$$
p(M)=\frac{N_{f}(M)}{N(M)}=\frac{a(M)}{H-a(M)},
$$

The energy content in a source volume is expressed by

$$
E=\frac{1}{2} e x^{2} V
$$

where $e$ denotes the elastic constant appropriate for the stress to which the medium within the source volume is subjected and $x$ represents the ultimate strain of the medium. $V$ is the volume of the source volume and is 


$$
V=\frac{4}{3} \pi a^{3} .
$$

If $e$ is taken to be $5 \times 10^{11}$ c.g.s. and $x$ to be $10^{-4}$, then $E(\mathrm{erg})$ is related to $a(\mathrm{~cm})$ as follows:

$$
E=10^{4} \times a^{3} .
$$

Combining eqs. (10) with (2), the following relation is obtained for connecting $a$ and $M$ :

$$
\log a(M)=2.6+0.5 M \text {. }
$$

The probability that an earthquake of magnitude $M$ is accompanied by surface fault appearance calculated by means of eqs. (9) and (11) is given in Table $I$, where $H$ is taken to be $40 \mathrm{~km}$.

Table I Probability of fault appearance

\begin{tabular}{c|r}
\hline$M$ & $p(M) \%$ \\
\hline 5.0 & 3 \\
5.5 & 6 \\
6.0 & 11 \\
6.2 & 14 \\
6.4 & 19 \\
6.6 & 25 \\
6.8 & 33 \\
7.0 & 46 \\
7.2 & 65 \\
7.4 & 100 \\
\hline
\end{tabular}

K. Hoshrno (1956) studied the relation between the earthquake magnitude and surface fault appearance in Japan and found that the percentage of the number $N_{f}(M)$ of earthquakes with faul appearance against the total number $N(M)$ of earthquakes of that magnitude is $60 \%$ for $7.0<M<7.4$ and $100 \%$ for $7.4<M$. IIDA also pointed out from world data that shallow earthquakes having magnitude greater than 7.3 are always accompanied by surface fault. Agreement of the calculated probability with the observed is satisfactory.

\section{$\S 4$. Lower limit of fault length}

Referring to Fig. 2 again, it is seen that, for earthquakes having $M$ greater than 7.4, a lower limit in fault length begins to appear as shown by the chain line. The six points representing extraordinarily long faults were not used in deriving eq. (4).

It $M$ exceeds 7.4 , the source volume can no longer be spherical, because the diameter of the sphere is then larger than the thickness of the earth's crust. The volume must be depressed or fiattened between the earth's surface and the bottom of the earth's crust in order to be big enough to store the energy needed for an earthquake of that magnitude, necessarily resulting in some fracture or fracture zone on the earth's surface. This will explain why the minimum fault length exists for earthquakes greater than 7.4 in magnitude, and at the same time, why extraoridinarily long faults sometimes appear accompanying these large earthquakes. That the spread in fault length suddenly decreases as $M=7.4$ is reached (Faults of zero length exist for $M$ less than 7.4, although they could not be shown in Fig. 2) also suggests the appropriateness of the above consideration.

\section{§ Summary and concluding remarks}

The observational relation between the fault length and the earthquake magnitude has been explained by the geometrical relation between the dimension of earthquake source volume with respect to the thickness of the earth's crust as schematically shown in the inset of Fig. 2. The observational fact has also been explained that the percentage of the number of earthquakes of a given magnitude accompanied by fault appearance against the total number of earthquakes of that magnitude increases from $60 \%$ for $7.0<M<7.4$ to $100 \%$ for $7.4<M$. Thus the iclea of voluminal storage of earthquake energy has been found to be useful here also.

In calculating the probability of fault appearance for an earthquake of a given magnitude, it has been assumed that earthquakes occur evenly through all clepths in the earth's crust. This however, is, not actually the case and the vertical distribution of earthquake origin is known to show a characteristic pattern, especially in Japan. If this is taken into account, the calculated probability 
of fault appearance based on the even distribution naturally undergoes change, although it is doubtful if the origin of an earthquake as determined by seismological observations should coincide with the centre of earthquake source volume.

IIDA (1963) pointed out that shallow submarine earthquakes having greater magnitude than 7.3 are always accompanied by Tsunamis. As already stated, he also pointed out that shallow earthquakes having greater magnitude than 7.3 are always accompanied by surface faults. The coincidence of these magnitude values must have an important bearing to the storage capacity of energy of the earth's crust material.

K. KaSAFARA $(1957,1958)$, in his study of the relation between geodetic and seismic aspects of earthquake phenomena, found that, in case of the Tango earthquake, Japan, of 1927, the characteristic period and push-pull distribution of seismic waves are satisfactorily explained by a spherical source model (Fig. 3). Kasahara adds to state that "stress change taking place on such a spherical surface can hardly be accepted from our

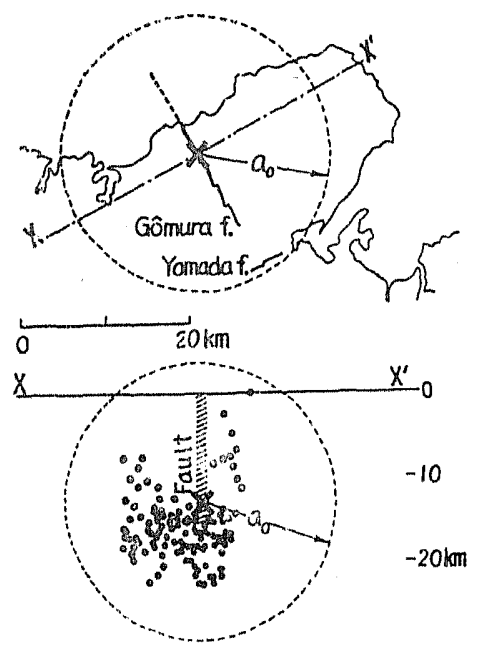

Fig. 3. Tango earthquake fault (1927). -and-.-: seismic faults, $x$ : origin of the main shock, aftershocks. The circle (broken lines) refers to the spherical model origin. (after K. KASAHARA (1958)). common experience of fracture production in a brittle substance, and furthermore, the model of a spherical origin is less suitable for understanding the most outstanding fact of remarkable shearing along a nodal line of push-pull distribution". But the present author is interested in the fact that the length of Gomura Fault accompanying the Tango earthquake happens to be approximately equal to the diameter of the circle defined as the intersection of the source sphere with the earth's surface. Kasarara studied the two dimensional problem of production of fault plane and elastic wave radiation from it and showed that the spectral intensity of $P$ wave is the maximum for the component wave of which the wave length is $1.2 \sim 1.5$ times that of fracture. That is

$$
\lambda_{8}=(1.2 \sim 1.5) \times 2 c,
$$

where $\lambda_{s}$ is the wave length of the component seismic wave which gives the maximum spectral intensity and $2 c$ is the length of fracture.

Now, if $c$ in eq. (12) is identified with the radius of the source sphere, and if the velocity of $\mathrm{P}$ wave in the medium is taken to be $5 \mathrm{~km} / \mathrm{sec}$, the following relation can be obtained:

$$
T^{*}=\frac{\lambda_{s}}{V}=\frac{(2.4 \sim 3.0) \times a}{5 \times 10^{5}},
$$

where $T^{*}$ is the characteristic period of $P$ wave. Taking the logarithm of both sides of eq. (13) and taking eq. (11) into account, the following relation is obtained for connecting $T^{*}$ and $M$ :

$$
\begin{aligned}
\log T^{*} & =-(5.22 \sim 5.32)+\log a \\
& =-(2.62 \sim 2.72)+0.5 M
\end{aligned}
$$

Eq. (14) agrees with the relation:

$$
\log T=-2.59+0.51 M
$$

given by KASAHARA by spectral analysis of seismic $P$ waves.

The coincidence of eq. (14) and eq. (15) is another evidence of appropriateness of the assumption that the fault length represents the diameter of the focal sphere. The author 
does not, however, insist that this must always be the case. In fact, if the fault must always extend up to the maximum dimension of outcrop area of the source volume, the plots in Fig. 2 must be more heavily concentrated around $L m$ than is the case in Fig. 2.

The most important assumption made in the above discussion is that the mantle of the earth does not participate in fracturing. No essential difference between the mechanism at the origin of crustal and subcrustal earthquakes has however been known and there is no reason why earthquake generating stress should not be accumulated in the earth's mantle. It must be admitted that the assumptions on which the preceding discussion has been developed are not more than simplifying working hypotheses for the time being.

\section{Reference}

Hoshino, K.:

1956 On Magnitude of Earthquakes Accompanied by Faults. Zisin (J. Seism. Soc. Japan), Ser. 2, 8, 160-162.
IIDA, K.:

1959 Earthquake energy and earthquake fault. J. Earth Sci., Nagoya Univ., đ, 98-107.

IIDA, K.:

1963 Magnitude of Tsunamigenic Earthquake, Aftershock Area, and Area of Tsunami Origin. Geophysical Papers Dedicated to Prof. Kenzo Sassa, 115-124.

Kasahara, K.:

1957 The Nature of Seismic Origins as Inferred from Seismological and Geodetic Observations (1). Bull. Earthq. Res. Inst. 35, 473532.

KASAHARA, K.:

1958 The Nature of Seismic Origins as Inferred from Seismological and Geodetic Observations (2). Bull. Earthq. Res. Inst. 36, 21-53.

TOCHER, D.:

1958 Earthquake Energy end Ground Breakage. Bull. Seism. Soc. Amer. 48, 147-153.

Tsuboi, C.:

1956 Earthquake Energy, Earthquake Volume, Aftershock Area, and Strength of the Earth's Crust. J. Phys. Earth, 4, 63-66.

Tsubor, C. and Otsuka, M.:

1963 Duration of Energy Radiation from an Earthquake Source Volume. Proc. Japan Acad., 39, 667-670. 